\title{
Vitamin A Metabolism: Challenges and Perspectives
}

\section{Ouliana Ziouzenkova*}

Department of Human Nutrition, Ohio State University, Columbus, Ohio, 43210, USA

\begin{abstract}
Vitamins set an unprecedented example of the essential environment-gene interactions that are necessary to sustain life. Seventeen Nobel prizes were awarded in the last century for discoveries related to vitamins' structures, physiology, and functions [1]. These discoveries help to abolish diseases related to vitamin deficiencies in the developed world and offer solutions to eradicate these disorders worldwide [2]. For example, embryonic malformations, night blindness, and immune deficiency in children were effectively treated by vitamin A supplementation $[2,3]$. In spite of encouraging results in vitamin-deficient subjects, wide applications of vitamin supplements in the nutrient-sufficient populations have been partially discouraging. Many clinical and supplementation trials reported increased mortality in subjects on long-term lipophilic vitamin $A$ and $E$ supplementations [4,5]. Especially striking deleterious effects were reported for lipophilic vitamin $A$ and provitamin $A$ ( $\beta$-carotene) $[4,6]$. The answer to safe application of lipophilic vitamins for the treatment and prevention of diseases may lie in the better understanding of their interaction with genes. There are two principal levels of lipophilic vitamin interactions with genes:
\end{abstract}

1) genes control metabolism of dietary vitamin into derivatives with hormone-like properties, and

2) vitamin-derived metabolites regulate specific gene programs through signaling and transcription pathways (Figure1).

Keywords: Retinaldehyde dehydrogenase; Raldh-1; Retinol; Abdominal fat; Alcohol dehydrogenases; Retinal

Abbreviations: ADH: Alcohol dehydrogenase; Aldh: Aldehyde
dehydrogenase; atRA: All-trans retinoic acid; ARAT: Acyl CoA-retinol
acyl transferase; BCMO1: $\beta$-Carotene 15,15'-carotene monooxygenase
1; BMP: Bone morphogenetic protein; $\beta$ LG: Beta-Lactoglobulin; C/
EBP: CCAAT enhancer-binding proteins; CRABP-II: Cellular retinoic
acid-binding protein II; CRBPIII: Cytosolic retinol-binding protein
type III (CRBPIII); DR1: Direct repeat motif 1, peroxisome proliferator-
activated receptor response element (PPRE); DR5: Direct repeat
motif 1, retinoic acid response element (RARE); EBF1: Early B-cell
factor; FABP: Fatty acid binding protein; HOX: Homeobox proteins;
HSL: Hormone-sensitive lipase; KLF: Krüppel-like transcription
factor; L-PGDS: Lipocalin-type prostaglandin (PG) D synthase; LPL:
Lipoprotein lipase; MAPK: p38 Mitogen-activated protein kinase;
NFATc1: Nuclear factor of activated T cells c1; PGC1a: PPAR
co-activator-1a; PML/RAR: Promyelocytic leukemia/retinoic acid
receptor alpha (a); PPARy: Peroxisome proliferator-activated receptor
$\gamma$; PPRE: Peroxisome proliferator-activated receptor response element
(DR1); pRb: Retinoblastoma protein; RAR: Retinoic acid receptor;
RARE: Retinoic acid response element (DR5); RA: Retinoic acid; Rald:
Retinaldehyde/retinal; Raldh: Retinaldehyde dehydrogenase; RBP:
Retinol-binding protein; RDH: Retinol dehydrogenases (microsomal);
SDR: Short-chain dehydrogenase/reductase; Shh: Sonic hedgehog; SR-
BI: Scavenger receptor class B type I; ZFP423: Zinc finger protein 423,
transcription factor

Introduction

Recent groundbreaking discoveries unveiled the complex interactions in the vitamin A pathway that underlie differentiation and metabolism in the majority of mammalian tissues during development and in adulthood. A summary of vitamin A metabolites and dependent genes regulating differentiation in some selected tissues is shown in (Figure 2). In this editorial, we briefly discuss some highlights and challenges in the understanding of vitamin A action. References will direct readers to the comprehensive reviews on these topics. Alphabetically-ordered abbreviations are provided in a list.

\section{Genes Participating in Vitamin A Metabolism}

Vitamin A is a generic term for retinol and retinyl esters present in the diet [7]. All metabolites emerging from these compounds are known as retinoids. Beta-carotene is defined as provitamin A because its central cleavage can potentially yield retinoids $[7,8]$. Metabolism of vitamin A results in the production of three major metabolites: retinol (ROH), retinaldehyde (retinal/Rald), and retinoic acid (RA) (Figure 1) [9]. In the course of vitamin $\mathrm{A} / \beta$-carotene metabolism, a broad range of other metabolites could be produced (e.g. dihydroretinol, 4-oxoRA and other oxidized RA derivatives, and apocarotenoids) $[7,8,10]$; however, their physiologic or therapeutic relevance await further investigation [11].

Vitamin A metabolism is encoded by genes participating in the absorption, uptake, transport (Figure 1, boxes), and enzymatic modification of retinoids, including major SDH/RDH and $\mathrm{ADH}$ families of Rald-generating enzymes and Aldh1 and AKR families [1216]. Dysregulation of these genes has been reported in association with cancerogenesis or differentiation in different tissues (some of them are outlined in Figure 2). The key roles of RDH10, Aldh1a2, and Cyp26 enzyme in embryogenesis were demonstrated in genetically deficient mice $[17,18]$. The embryonic lethal phenotype in these mice limits studies on the possible causal role of these genes in tissue differentiation in adulthood. Currently, the role of vitamin A metabolizing enzymes is studied in greater detail during embryogenesis. The emerging studies on differentiation during in adulthood underscore the role of Aldh1a2-dependent RA production in antigen presentation [19], of Aldh1 enzymes in the formation of fat depots [20], RBP4/STRA6 in adipogenesis and insulin sensitivity [21], DHRS9, Aldh1, CRABP2, and

*Corresponding author: Ouliana Ziouzenkova PhD, Assistant Professor Department Of Human Nutrition, Ohio State University, 1787 Neil Avenue, 331A Campbell Hall, Columbus, OH 43210, USA, Tel: 614292 5034; Fax: 614292 8880; E-mail: ziouzenkova.1@osu.edu

Received January 20, 2012; Accepted January 20, 2012; Published January 25 2012

Citation: Ziouzenkova O (2012) Vitamin A Metabolism: Challenges and Perspectives. Vitam Trace Elem 1:e106. doi:10.4172/2167-0390.1000e106

Copyright: @ 2012 Ziouzenkova O. This is an open-access article distributed under the terms of the Creative Commons Attribution License, which permits unrestricted use, distribution, and reproduction in any medium, provided the original author and source are credited. 


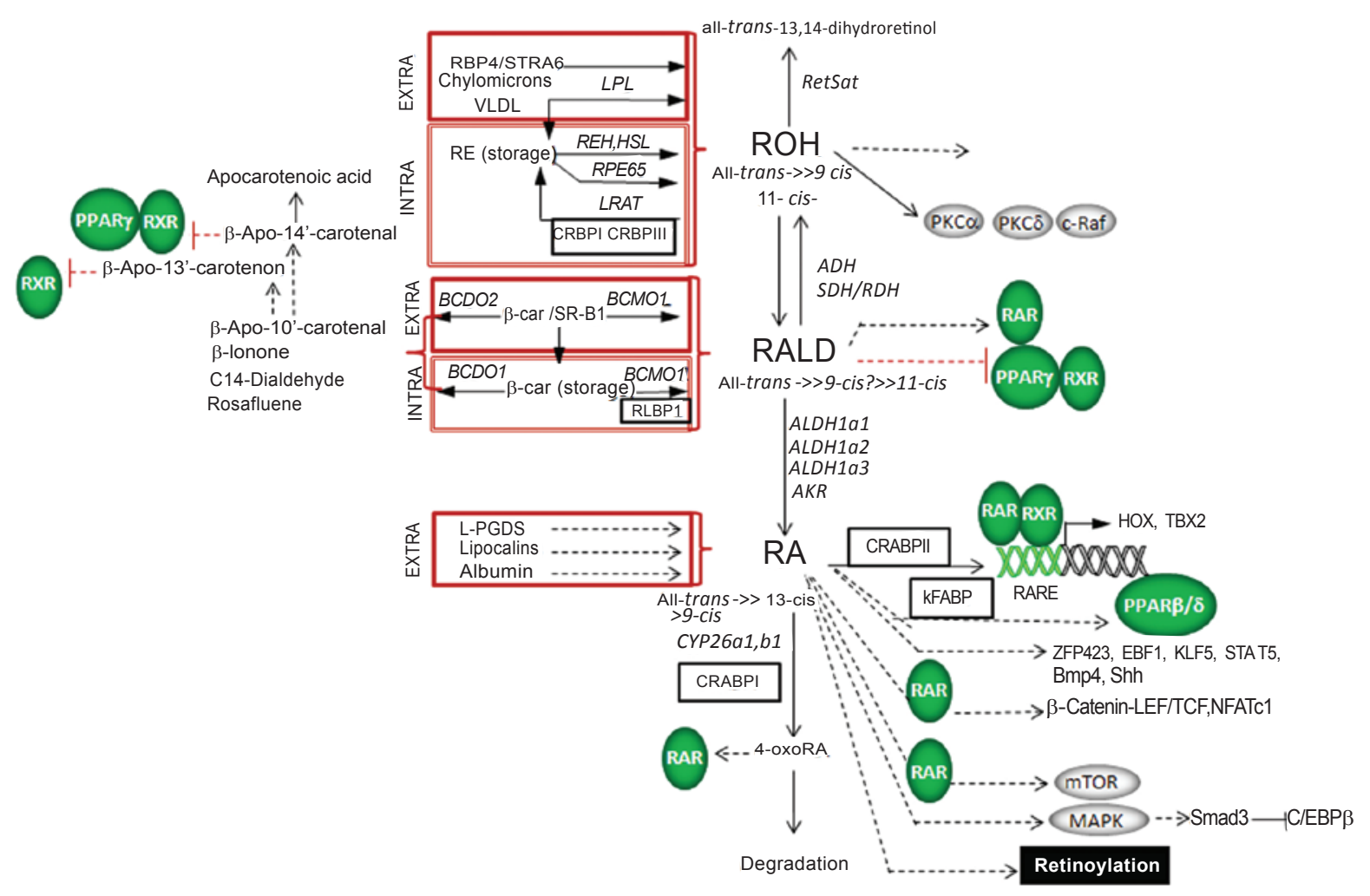

Figure 1: Principal components of cellular vitamin A metabolism.

Red boxes indicate cellular transport mechanisms from blood (Extra) or intracellular storage (Intra). Enzymes are shown in italic font, binding proteins are in black boxes. Dashed lines indicate emerging links that are not completely understood. Examples of critical transcription and signaling pathways activated by retinoids are indicated on the right side. Nuclear receptors are shown in green ovals in contrast to other transcription factors. Key kinases are shown in gray ovals. RA's role in chemical protein modification, known as retinoylation, is indicated in the solid black box. All abbreviations are shown in the list.

The STRA6 receptor mediates uptake of retinol bound to the retinol-binding protein (RBP4). CRBPI binds retinol, while LRAT converts retinol to retinyl esters for storage in lipid droplets. Lipoprotein lipase (LPL) mediates hydrolysis of retinyl esters (RE) and retinol from chylomicron remnants. Retinol can be transported intracellularly by CRBPI and/or CRBPIII. Hormone sensitive lipase (HSL) is the major retinyl ester hydrolase in adipose tissue. Less explored pathways: $\beta$-carotene, transported in HDL, could be taken up by the SR-B1 receptor. In cells, symmetric cleavage mediated by BCMO1 leads to the production of two retinaldehyde (Rald) molecules, whereas asymmetric cleavage by BCDO2 generates various apocarotenals and their oxidation products (few major derivatives are indicated). RA could be delivered from blood bound to lipocalins and albumin.

Major metabolites are retinol $(\mathrm{ROH})$, retinaldehyde (retinal, Rald), and retinoic acid (RA). ROH can undergo 1) reversible oxidation to retinaldehyde by either alcohol dehydrogenase $(\mathrm{ADH})$ or retinol dehydrogenase/reductase $(\mathrm{RDH})$ families of enzymes, 2) esterification into RE, or 3) saturation by RetSat, generating all-trans-13,14dihydroretinol and, in further enzymatic reactions, dihydroretinoids. $\mathrm{ROH}$ is a weak agonist for RAR, but it regulates PKC and c-raf.

Rald can be irreversibly oxidized to RA by ALDH1 and aldo-keto reductase (Akr) families of enzymes or reduced to retinol by SDR/RDH families of enzymes, respectively. Rald also binds to CRBPI in vitro; however, it is unknown if this protein can transport Rald to the nucleus. In the eye, Rald is transported by RLBP1. Rald is a weak agonist for RAR and can repress PPARY and RXRa.

The cytosolic aldehyde dehydrogenase 1 (ALDH1) family of enzymes is comprised of three members (Aldh1a1, Aldh1a2, and Aldh1a3, known also as Raldh1, Raldh2, and Raldh3). Aldh1 is the major RA-generating family of enzymes. The produced RA can act in an autocrine fashion in adipocytes and in a paracrine fashion in embryogenesis. RA-binding protein II (CRABPII) facilitates RA transport to the nucleus where RA binds to RAR. RA binding to kFABP appears to activate the PPAR $\beta / \delta$ transcription factor. Activated RAR can heterodimerize with RXR and regulate target genes or other transcription factors (HOX family members, TBX2, and others) that contain RAR response element (RARE). RA can also act through other transcription factors through unidentified actions of RAR or by unknown mechanisms. Similarly, signaling pathway (MAPK, mTOR) can occur in an RAR dependent manner or by unidentified mechanisms. CRABPI appears to deliver RA for degradation by the cytochrome P450 (CYP26) family of enzymes.

CRABPII in hair follicles/sebaceous glands [22], RDH, ADH, Aldh1 in stem cell differentiation [23,24], ADH/Aldh1/AKR in cancerogenesis $[25,26]$, and other differentiation and metabolic processes. The translational potential of these findings remains to be elucidated.

Studies on vitamin A-metabolizing enzymes are notoriously challenging. Hydrolysis of ROH from RE, generation of Rald from $\mathrm{ROH}$, and RA from Rald (Figure 1) are mediated by a family of enzymes that function redundantly [12,27]. Therefore, the function of each enzyme needs to be analyzed in conjunction with the presence and activity of other enzymes from this family as well as enzymes providing substrates (e.g. Rald generation for RA synthesis by Aldhla1, a2, and a3 enzymes). Although the enzymes from the same family have common enzymatic actions, many of them exert specific functions. For instance, the Aldh1a1 enzyme can utilize other substrates if they are present at high concentrations. In contrast, Aldh1a2 and a3 enzymes utilize only Rald as a substrate $[28,29]$. The presence of other substrates for Aldh1a1 may potentially alter the balance of retinoid production.

Vitamin A-metabolizing enzymes are expressed in a tissue- and/ 


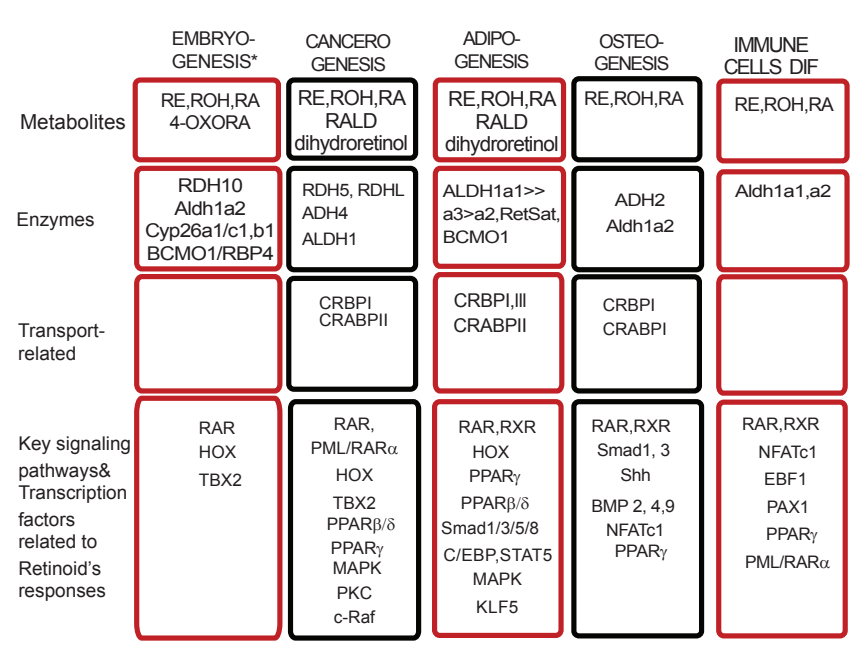

*Embryonic lethal phenotype in null mutants or double knockout mice ( such as Cyp26a1/c1 and BCMO1/RBP4).All factors depicted in Fig.1influence organogenesis of specific tissues during the development.

Figure 2: Examples of differentiation processes requiring vitamin A metabolism.

or sex-specific manner; however, the functional relevance of such expression is understudied. Recently, we showed that fat formation in detrimental visceral vs. unharmful subcutaneous fat depots is determined by the fat-depot-specific expression of Aldh1a1 and Aldh1a3, which produce depot-specific levels of RA for adipogenesis [20]. Whether the changes in Aldh1 enzymes also influence divergent properties of visceral and subcutaneous fat remains has to be investigated.

Redox properties of retinoids are another challenge hindering the investigation of vitamin A-related pathways. The majority of retinoids, but especially intermediate metabolites like Rald, undergo rapid reduction to $\mathrm{ROH}$ or oxidation to RA (Figure1). Moreover, the conversion rate may depend on the redox status in the cells, since all $\mathrm{ADH}, \mathrm{RDH} / \mathrm{SDH}$, and Aldh1 enzymes are $\mathrm{NAD}^{+} / \mathrm{NADH}$ - or NADPdependent [16]. It is expected that several retinoid metabolites, such as $\mathrm{ROH}$, Rald, and RA are present in the form of various isomers (cis and trans) simultaneously at different concentrations in a cell. In addition to enzymatic modifications, retinoids are prone to oxidation, which can be induced by light and handling [30]. These properties, low concentrations in tissues, and high lipophilicity of retinoids, leave quantitative analysis of these compounds in tissues a prerogative of few specialized laboratories [30]. Quantification of 9-cis RA in pancreas and brain in the Napoli laboratory led to the astounding findings of the RA's role in insulin production and brain function [31]. It is worth notice that trans and cis isomers of retinoids act through different molecular mechanisms, and generation of 9-cis RA in the cell is still a subject of debate [32].

Retinoids participate in their own regulation. In adipocytes, the addition of RA to the cells leads to a dose-dependent inhibition of Aldh1a1 expression by a negative feedback mechanism [33]. However, this regulation could differ in other tissues and be gender-dependent. In fact, many vitamin A metabolizing enzymes are expressed in a tissue-specific manner in males and females [34] by marginally understood mechanisms. Aldh1a2 expression appears to be regulated directly by estrogen receptor in its promoter region [35], whereas other Aldh1 enzyme levels are sensitive to sex-hormones by other, undefined mechanisms [36]. The sex-specific expression of RBP4 is linked to different levels of leptin in males and females [34]. Many mice genetically deficient in vitamin A metabolizing enzymes exhibit pronounced sex differences [37]. Undoubtedly, vitamin A metabolism in men and women may determine their responses to nutrients, vitamin supplements, and retinoid therapies. Understanding of vitamin A metabolizing gene regulation is a necessary step for the development of personalized, safe, and effective therapies with retinoids.

\section{Retinoid-dependent Regulation of Specific Signaling and Transcriptional Pathways}

The major breakthrough in the molecular mechanisms of retinoid action came from the Dr. Chambon's laboratory, reporting the discovery of the retinoic acid receptor (RAR) in 1987 [38], followed by identification of its heterodimerization partner RXR [39]. These receptors act as transcription factors upon binding of RA [40,41]. All RA isomers, 4-oxo RA, ROH, and Rald, can activate RAR isoforms [42]; 9-cis RA activates RXR [39], and all-trans RA activates PPAR $\beta / \delta$ transcription factors [43]. Figure 1 depicts examples of multiple other transcription factors that are under control of RAR [40]. Many transcription factors, such as ZFP423, are RA-sensitive, by yet unknown mechanisms of action [33].

Cytosolic action of RAR has also been revealed. RAR appears to participate in the activation of critical signaling pathways, such as mTOR, and protein translation, at least in the neurons $[40,44]$. These findings raise important questions about partition of RA between cytosolic and nuclear compartments or among different transcription factors dependent on RA. The answer may lie in the binding proteins. Dr. Noy's laboratory showed that RA binding to CRABPII activates RAR, whereas expression of $k F A B P$ leads to activation of PPAR $\beta / \delta$ transcription factor [45]. Obviously, the specificity of RA responses in different tissues will also depend on the expression of different transcription factors. The dynamic changes in transcription factors during differentiation, could, potentially, alter responses to RA in the same experimental system, such as adipogenesis in 3T3-L1, which can be inhibited or facilitated by RA [20]. In adipogenesis, RA responses are also markedly influenced by RA concentration, circadian cycle, and the presence of metabolizing enzymes. Debates about RA effects in obesity continue, because vitamin A supplementation/RA administration can promote obesity or weight loss in different experimental models $[20,46]$. Often these experiments do not account for endogenous vitamin A metabolism and mobilization of retinoids from internal storage. RA can also mediate paracrine effects in tandem with other transcription factors: RA induces HOX transcription factors that in turn regulate RAR and Aldh1 expression in organogenesis [9]. More studies are needed to establish the hierarchy in transcription factor activation by RA [20]. Chromosome-wide studies of hormone actions led to the surprising conclusion that regulation of promoters occurred only in a minor portion of hormone-sensitive genes [47]. Such studies have not been performed for RA. Even less is known about RA epigenetic effects. Comprehensive studies on RA action will unravel molecular targets for RA responsible for the therapeutic effects and toxicity of this potent metabolite.

Molecular targets of metabolites other than RA are nearly unknown. The emerging examination of these pathways has led to fruitful findings. $\mathrm{ROH}$ action on PKC appears to regulate cancerogenesis [48]. Rald and apocarotenoids may participate in the inhibition of transcription factors RXR ad PPAR $\gamma$ and, thereby, regulate fat formation [11]. Dihydroretinoids, 4-oxo RA, and other oxidative RA derivatives, $\beta$-10'apocarotenal and $\beta$-ionone are produced by specific enzymes (Figure 1 ) 
and have been detected in vivo [49], however, their specific molecular targets remained to be discovered.

\section{Conclusion}

Possibly, vitamin A regulates the broadest range of gene programs in mammalian development, tissue differentiation, and metabolism (Figure 1). Incomplete understanding of genes participating in vitamin A metabolism and retinoid interactions with signaling and transcription pathways is a major drawback in the safe application of vitamin A. OMICS open access journal, 'Vitamins \& Trace Elements' will foster reports, discussions, opinions, and reviews to facilitate the development of the vitamin A research field.

\section{References}

1. Carpenter K (2012) The Nobel Prize and the Discovery of Vitamins.

2. Imdad A, Yakoob MY, Sudfeld C, Haider BA, Black RE, et al. (2011) Impact of vitamin A supplementation on infant and childhood mortality. BMC Public Health 11 Suppl 3: S20.

3. Mayo-Wilson E, Imdad A, Herzer K, Yakoob MY, Bhutta ZA (2011) Vitamin A supplements for preventing mortality, illness, and blindness in children aged under 5: systematic review and meta-analysis. BMJ.

4. Bjelakovic G, Nikolova D, Gluud LL, Simonetti RG, Gluud C (2007) Mortality in randomized trials of antioxidant supplements for primary and secondary prevention: systematic review and meta-analysis. JAMA 297: 842-857.

5. Dotan Y, Pinchuk I, Lichtenberg D, Leshno M (2009) Decision analysis supports the paradigm that indiscriminate supplementation of vitamin $\mathrm{E}$ does more harm than good. Arterioscler Thromb Vasc Biol 29: 1304-1309.

6. Jeon YJ, Myung SK, Lee EH, Kim Y, Chang YJ, et al. (2011) Effects of Betacarotene supplements on cancer prevention: meta-analysis of randomized controlled trials. Nutr Cancer 63: 1196-1207.

7. Krinsky N, Russell RM (2001) Regarding the conversion of beta-carotene to vitamin A. Nutr Rev 59: 309.

8. von Lintig J (2012) Metabolism of carotenoids and retinoids related to vision. $J$ Biol Chem 287: 1627-1634.

9. Duester G (2008) Retinoic acid synthesis and signaling during early organogenesis. Cell 134: 921-931.

10. Muindi JR, Young CW, Warrell RP Jr (1994) Clinical pharmacology of all-trans retinoic acid. Leukemia $\quad 8: 1807-1812$.

11. Ziouzenkova O, PlutzkyJ (2008) Retinoid metabolism and nuclear receptor responses: New insights into coordinated regulation of the PPAR-RXR complex. FEBS Lett 582: 32-38.

12. Harrison $\mathrm{EH}$ (2012) Mechanisms involved in the intestinal absorption of dietary vitamin A and provitamin A carotenoids. Biochim Biophys Acta 1821: 70-77.

13. ShirakamiY, Lee SA, Clugston RD, Blaner WS (2012) Hepatic metabolism of retinoids and disease associations. Biochim Biophys Acta 1821: 124-136.

14. Paik J, Vogel S, Quadro L, Piantedosi R, Gottesman M, et al. (2004) Vitamin A: overlapping delivery pathways to tissues from the circulation. J Nutr 134 276S-280S.

15. Sun $H$ (2012) Membrane receptors and transporters involved in the function and transport of vitamin A and its derivatives. Biochim Biophys Acta 1821: 99112.

16. Duester G, Mic FA, Molotkov A (2003) Cytosolic retinoid dehydrogenases govern ubiquitous metabolism of retinol to retinaldehyde followed by tissuespecific metabolism to retinoic acid. Chem Biol Interact 143-144: 201-210.

17. Kumar S, Sandell LL, Trainor PA, Koentgen F, Duester G (2012) Alcohol and aldehyde dehydrogenases: Retinoid metabolic effects in mouse knockout models. Biochim Biophys Acta 1821: 198-205.

18. Ross AC, Zolfaghari R (2011) Cytochrome P450s in the regulation of cellular retinoic acid metabolism. Annu Rev Nutr 31: 65-87.

19. Duriancik DM, Lackey DE, Hoag KA (2011) Vitamin A as a regulator of antigen presenting cells. J Nutr 140: 1395-1399.

20. Yasmeen R, Jeyakumar SM, Reichert B, Yang F, Ziouzenkova O (2012) The contribution of vitamin A to autocrine regulation of fat depots. Biochim Biophys Acta 1821: 190-197.

21. Berry DC, Noy N (2012) Signaling by vitamin A and retinol-binding protein in regulation of insulin responses and lipid homeostasis. Biochim Biophys Acta 1821: $168-176$

22. Everts HB (2012) Endogenous retinoids in the hair follicle and sebaceous gland. Biochim Biophys Acta 1821: 222-229.

23. Gasparetto M, Sekulovic S, Brocker C, Tang P, Zakaryan A, et al. (2011) Aldehyde dehydrogenases are regulators of hematopoietic stem cell numbers and B-cell development. Exp Hematol.

24. Gudas LJ, Wagner JA (2011) Retinoids regulate stem cell differentiation. J Cell Physiol 226: 322-330.

25. Jelski W, Szmitkowski M (2008) Alcohol dehydrogenase (ADH) and aldehyde dehydrogenase (ALDH) in the cancer diseases. Clin Chim Acta 395: 1-5.

26. Ruiz FX, Gallego O, Ardevol A, Moro A, Alvarez S, et al. (2009) Aldo-keto reductases from the AKR1B subfamily: retinoid specificity and control of cellular retinoic acid levels. Chem Biol Interact 178: 171-177.

27. Napoli JL (2012) Physiological insights into all-trans-retinoic acid biosynthesis Biochim Biophys Acta 1821: 152-167.

28. Kong D, Kotraiah V (2011) Modulation of Aldehyde Dehydrogenase Activity Affects (+/-)-4-Hydroxy-2E-nonenal (HNE) Toxicity and HNE-Protein Adduct Levels in PC12 Cells. J Mol Neurosci.

29. Makia NL, Bojang P, Falkner KC, Conklin DJ, Prough RA (2011) Murine hepatic aldehyde dehydrogenase $1 \mathrm{a} 1$ is a major contributor to oxidation of aldehydes formed by lipid peroxidation. Chem Biol Interact 191: 278-287.

30. Kane MA (2012) Analysis, occurrence, and function of 9-cis-retinoic acid Biochim Biophys Acta 1821: 10-20.

31. Kane MA, Folias AE, Pingitore A, Perri M, Obrochta KM, et al. (2010) Identification of 9-cis-retinoic acid as a pancreas-specific autacoid that attenuates glucose-stimulated insulin secretion. Proc Natl Acad Sci U S A 107 21884-21889.

32. Mic FA, Molotko A, Benbrook DM, Duester G (2003) Retinoid activation of retinoic acid receptor but not retinoid $X$ receptor is sufficient to rescue letha defect in retinoic acid synthesis. Proc Natl Acad Sci U S A 100: 7135-7140.

33. Reichert B, Yasmeen R, Jeyakumar SM, Yang F, Alder H, et al. (2011) Concerted action of aldehyde dehydrogenases influences depot-specific fat formation. Mol Endocrinol.

34. Kos K, Wong S, Tan BK, Kerrigan D, Randeva HS, et al. (2010) Human RBP4 adipose tissue expression is gender specific and influenced by leptin. Clin Endocrinol (Oxf) 74: 197-205

35. Wang X, Sperkova Z, Napoli JL (2001) Analysis of mouse retinal dehydrogenase type 2 promoter and expression. Genomics 74: $245-250$

36. Trasino SE, Harrison EH, Wang TT (2007) Androgen regulation of aldehyde dehydrogenase $1 \mathrm{~A} 3(\mathrm{ALDH} 1 \mathrm{~A} 3)$ in the androgen-responsive human prostate cancer cell line LNCaP. Exp Biol Med (Maywood) 232: 762-771.

37. van Helden YG, Godschalk RW, von Lintig J, Lietz G, Landrier JF, et al. (2011) Gene expression response of mouse lung, liver and white adipose tissue to $\beta$-carotene supplementation, knockout of Bcmo1 and sex. Mol Nutr Food Res 55: $1466-1474$

38. Petkovich M, Brand NJ, Krust A, Chambon P (1987) A human retinoic acid receptor which belongs to the family of nuclear receptors. Nature 330: 444-450.

39. Heyman RA, Mangelsdorf DJ, Dyck JA, Stein RB, Evans RM, et al. (1992) 9-cis retinoic acid is a high affinity ligand for the retinoid $X$ receptor. Cell 68: 397-406.

40. Germain P, Chambon P, Eichele G, Evans RM, Lazar MA, et al. (2006) International Union of Pharmacology. LX. Retinoic acid receptors. Pharmacol Rev 58: 712-725.

41. Germain P, Chambon P, Eichele G, Evans RM, Lazar MA, et al. (2006) International Union of Pharmacology. LXIII. Retinoid X receptors. Pharmaco Rev 58: 760-772.

42. Repa JJ, Hanson KK, Clagett-Dame M (1993) All-trans-retinol is a ligand for the retinoic acid receptors. Proc Natl Acad Sci U S A 90: 7293-7297.

43. Shaw N, Elholm M, Noy N (2003) Retinoic acid is a high affinity selective ligand for the peroxisome proliferator-activated receptor beta/delta. J Biol Chem 278 : 41589-41592. 
44. Chen N, Napoli JL (2008) All-trans-retinoic acid stimulates translation and induces spine formation in hippocampal neurons through a membraneassociated RARalpha. FASEB J 22: 236-245.

45. Schug TT, Berry DC, Shaw NS, Travis SN, Noy N (2007) Opposing effects of retinoic acid on cell growth result from alternate activation of two different nuclear receptors. Cell 129: 723-733.

46. Bonet ML, Ribot J, Palou A (2012) Lipid metabolism in mammalian tissues and its control by retinoic acid. Biochim Biophys Acta 1821: 177-189.
47. Carroll JS, Liu XS, Brodsky AS, Li W, Meyer CA, et al. (2005) Chromosomewide mapping of estrogen receptor binding reveals long-range regulation requiring the forkhead protein FoxA1. Cell 122: 33-43.

48. Hoyos B, Acin-Perez R, Fischman DA, Manfredi G, Hammerling U (2012) Hiding in plain sight: Uncovering a new function of vitamin $A$ in redox signaling Biochim Biophys Acta 1821: 241-247.

49. Lobo GP, Amengual J, Palczewski G, Babino D, von Lintig J (2012) Mammalian Carotenoid-oxygenases: Key players for carotenoid function and homeostasis. Biochim Biophys Acta 1821: 78-87. 